\title{
UN ANÁLISIS DE LA REGULACIÓN ANDALUZA DE SECCIONES DE CRÉDITO
}

\author{
POR \\ Amalia HIDALGO-FERNÁNDEZ ${ }^{1}$ y \\ Francisco Javier BERNABÉU AGUILERA ${ }^{2}$
}

\section{RESUMEN}

Las secciones de crédito se encuentran inicialmente reguladas en la normativa cooperativa estatal, no obstante, queda supeditada su regulación a la normativa autonómica. No todas las comunidades autónomas han avanzado al mismo tiempo y en la misma dirección en la regulación de las secciones de crédito, de manera que pueden identificarse distintos modelos atendiendo a una normativa, que podríamos calificar como favorable, desfavorable o neutra en relación con su regulación. El objetivo de este trabajo es analizar el contenido de la nueva normativa prevista para las cooperativas andaluzas con sección de crédito, la cual se presenta como una garantía y oportunidad al plantear determinadas estrategias de control y gestión que consigan dotar a las secciones de crédito de unos elementos que les permitan intervenir en el sistema financiero de una manera eficiente y a su vez, someterlas a unos niveles de control adecuados a sus características.

Palabras Clave: cooperativas, financiación, gestión, legislación autonómica, secciones de crédito crisis sectorial.

Claves Econlit: G000, G010, M100, P130

\footnotetext{
${ }^{1}$ Profesora Doctora. Departamento de Economía, Sociología y Política Agrarias. Universidad de Córdoba. Dirección de correo electrónico: ahidalgo@uco.es

${ }^{2}$ Profesor Asociado. Departamento de Economía, Sociología y Política Agrarias. Universidad de Córdoba. Dirección de correo electrónico: jba@audiel.es
}

REVESCO No 114 - Primer Cuatrimestre 2014 - ISSN: 1885-8031 - www.ucm.es/info/revesco

http://dx.doi.org/10.5209/rev_REVE.2014.v114.44289

Fecha de recepción: 15/04/2013

Fecha de aceptación: 04/07/2013 


\title{
AN ANALYSIS OF THE REGULATION OF CREDIT SECTIONS ANDALUZAS
}

\begin{abstract}
The credit sections are initially regulated by state cooperative legislation, however, their regulation is subject to the regional rules. Not all regions have increased at the same time and in the same direction in regulating credit sections so that different models can be identified pursuant to a statute, we could describe as favorable, unfavorable or neutral in relation to its regulation. The aim of this paper is to analyze the content of the new rules provided for Andalucía cooperatives with credit section, which is presented as a security and opportunity to raise certain control and management strategies that achieve sections provide a credit elements that allow them to intervene in the financial system in an efficient manner and in turn, submit them to control levels appropriate to their characteristics.
\end{abstract}

Keywords: Cooperatives, finance, management, regional legislation, credit sections.

\section{INTRODUCCIÓN}

El sistema financiero se puede definir como el conjunto de instituciones, mercados y activos que tienen por finalidad canalizar los superávit de recursos desde unas unidades económicas a otras de déficit, lo que lleva implícito la existencia de relaciones financieras entre los intervinientes con la finalidad de conseguir los objetivos de los mismos. A estos efectos, se identifican como entidades de crédito:

- El Instituto de Crédito Oficial.

- Los Bancos.

- Las Cajas de Ahorros y la Confederación Española de Cajas de Ahorros.

- $\quad$ Las Cooperativas de Crédito.

- Los Establecimientos Financieros de Crédito.

El concepto de entidad de crédito se recoge en la normativa financiera, en la Ley 26/1988, de 29 de julio, de Disciplina e Intervención de las Entidades de Crédito ${ }^{3}$, según la cual se consideran entidades de crédito las enumeradas en el apartado segundo del artículo 1 del Real Decreto Legislativo 1298/1986, de 28 de junio, sobre adaptación del Derecho vigente

${ }^{3}$ Artículo 1.2 de la citada Ley

REVESCO No 114 - Primer Cuatrimestre 2014 - ISSN: 1885-8031 - www.ucm.es/info/revesco 
en materia de entidades de crédito al de las Comunidades Europeas (en la redacción dada por la Disposición Final primera de la Ley 21/2011, de 26 de julio, de dinero electrónico), que establece que, de acuerdo con la Directiva 2000/12/CE del Parlamento Europeo y del Consejo, de 20 de marzo de 2000, relativa al acceso a la actividad de las entidades de crédito y a su ejercicio, se entiende por «entidad de crédito» toda empresa que tenga como actividad típica y habitual recibir fondos del público en forma de depósito, préstamo, cesión temporal de activos financieros u otras análogas que lleven aparejada la obligación de su restitución, aplicándolos por cuenta propia a la concesión de créditos u operaciones de análoga naturaleza, correspondiendo al Banco de España las competencias regulatorias.

Dentro de esta definición del sistema financiero no pueden incluirse las secciones de crédito constituidas en el seno de las cooperativas, aunque realicen servicios de intermediación financiera similares a los ofrecidos por las entidades de crédito, encontrándose reguladas en la normativa específica sobre cooperativas contenida en la Ley 27/1999, de 16 de julio, General de Cooperativas, que en su artículo 5 establece que su regulación queda supedita a la normativa autonómica. A efectos de que no pueda producirse una "confusión" entre la prestación de servicios de intermediación permitidos a las secciones de crédito y otros componentes del sistema financiero, se prohíbe que la cooperativa pueda incluir en su denominación las expresiones "cooperativa de crédito", "caja rural" u otra análoga, ni sus abreviaturas.

En esta regulación autonómica (Campos y Sanchís, 2005), no todas las comunidades han avanzado en la misma dirección, de manera que pueden identificarse tres modelos atendiendo a una normativa favorable, desfavorable, restrictiva y neutra en relación con la regulación específica de las secciones de crédito, según se recoge en el siguiente cuadro 1:

Cuadro 1. Posición de las normativas autonómicas ante las secciones de crédito

\begin{tabular}{|c|c|c|}
\hline Comunidad & Normativa & Posición \\
\hline Valencia & $\begin{array}{c}\text { DECRETO } 151 / 1986, \text { de } 9 \text { de diciembre, por el que se } \\
\text { desarrolla la Ley } 8 / 1995 .\end{array}$ & Desfavorable \\
\hline Extremadura & DECRETO 129/2002, de 24 de septiembre, & Poco favorable \\
\hline Cataluña & $\begin{array}{c}\text { DECRETO 280/2003, de 4 de noviembre, de desarrollo de la } \\
\text { Ley 6/1998, de 13 de mayo. }\end{array}$ & Favorable \\
\hline Andalucía & Ley $14 / 2011$, de 23 de diciembre & Sin regulación \\
\hline
\end{tabular}

REVESCO No 114 - Primer Cuatrimestre 2014 - ISSN: 1885-8031 - www.ucm.es/info/revesco 
\begin{tabular}{|c|c|c|}
$\begin{array}{c}\text { Resto } \\
\text { comunidades }\end{array}$ & Ley 27/1999, general de cooperativas & Neutra \\
\hline
\end{tabular}

Fuente: Elaboración propia

Debe entenderse como una posición favorable aquella que favorece el nacimiento y consolidación de las secciones de crédito en el ámbito cooperativo, estableciendo un marco normativo que no limite ni el número o volumen de operaciones, ni aquellos que puedan relacionarse con la sección.

A pesar del diferente tratamiento que se da a estas secciones en la normativa cooperativa, todas ellas tienen en común una definición similar de las mismas, organización y funcionamiento:

1) No tienen personalidad jurídica propia y se constituyen en el seno de la cooperativa.

2) Debe recogerse su constitución en los estatutos de la cooperativa.

3) Actúan como intermediarios financieros.

4) La actividad de las secciones de crédito no puede ser la principal de la entidad.

5) Mantienen contabilidad separada y su gestión debe ser independiente del resto de secciones.

Los aspectos más destacables de la regulación prevista en la normativa andaluza, se centran en los aspectos económicos y financieros de las secciones de crédito y ante este escenario, el objetivo del trabajo es analizar el efecto de la nueva normativa sobre las secciones de crédito andaluzas, comparando la misma con la normativa en las comunidades autónomas más significativas.

\section{NATURALEZA Y FINALIDAD DE LAS SECCIONES DE CRÉDITO.}

Las secciones de crédito son, como su propio nombre indica, una sección creada dentro de la cooperativa, carentes de personalidad jurídica, y cuya finalidad es realizar las actividades propias de las entidades financieras en el seno de la cooperativa, con el objetivo básico de satisfacer las necesidades de socios y otras secciones de la cooperativa. La legislación permite la creación de secciones de crédito en todos los tipos de cooperativas, sólo las excluye explícitamente en las cooperativas de crédito por su propia naturaleza financiera, habiéndose desarrollado considerablemente en el sector de las cooperativas agrarias. 
Las sociedades cooperativas agrarias presentan una estructura diferente al resto de las empresas, al inclinarse por una administración dividida en secciones resultado de un proceso de agrupación de actividades (Bel, 2000) por lo que las secciones de crédito encuentran en éstas un espacio muy favorable para su florecimiento

A través de la sección de crédito los socios participan en el mantenimiento de una estructura de pasivo sólida en la cooperativa, actuando como proveedor de recursos cuando realiza un depósito, y como cliente cuando demanda un crédito. Esta actividad se extiende a asociados, familiares directos, trabajadores e incluso ex-socios, dependiendo de dónde se localice la cooperativa, de manera que se genera un amplio abanico de actividad que posibilita la consecución de los objetivos de la sociedad cooperativa con el apoyo de la actividad desarrollada a través de la Sección de Crédito.

En este sentido, es importante destacar que la finalidad de las secciones de crédito, se puede resumir en la contribución a la consecución del objeto social de la cooperativa, concretándose en las siguientes facetas:

a) Contribuir a la financiación de las operaciones de la sociedad.

b) Contribuir a la financiación de las actividades de las personas socias vinculadas a la actividad de la sociedad.

c) Gestionar de manera conjunta las disponibilidades líquidas de las personas socias y de la propia entidad.

\section{ACTIVIDAD DESARROLLADA POR LA SECCIÓN DE CRÉDITO}

La distinta normativa (Melián, 2005) que regula las secciones de crédito, atendiendo a la comunidad autónoma en que se desarrollen, determina si los intervinientes en la actividad financiera de la sección de crédito pueden ser exclusivamente socios y secciones, o puede extenderse a asociados, trabajadores, familiares directos e incluso ex-socios. En cualquiera de los casos, las relaciones básicas mantenidas por éstos con la sección de crédito, se pueden representar gráficamente como se describe a continuación en el Gráfico 1.

Se observa que las secciones de crédito actúan, como canalizadoras del ahorro desde los socios, asociados, trabajadores, ex-socios, etc. a las secciones de la cooperativa y a otros socios, asociados...., cumplen la finalidad de contribución a la financiación de los elementos intervinientes y, en definitiva, contribuyen al objeto social de la cooperativa. 
Las secciones de crédito se constituyen por tanto, en verdaderos intermediarios financieros entre los excedentes a disposición de los socios ahorradores (asociados, familiares, etc.) y el resto de socios y secciones de la propia cooperativa, canalizando los ahorros de los primeros hacia los segundos.

La finalidad y justificación de las secciones de crédito se encuentra tanto en servir de instrumento financiero a los socios y resto de secciones de la cooperativa, como constituirse en una opción estratégica de la cooperativa frente a otras opciones de financiación menos ventajosas. La sección de crédito especializada en la actividad financiera de la cooperativa, no intenta variar el objeto social de la misma, aunque sus objetivos sean la máxima rentabilidad económica y/o financiera de las aportaciones de los socios a la sociedad, bien por su participación en la actividad de bienes y/o prestación de servicios o por su participación en el proceso financiero (García-Gutiérrez, 1992; Campos y Sanchís, 2005).

Gráfico 1. Relaciones básicas de las secciones de crédito

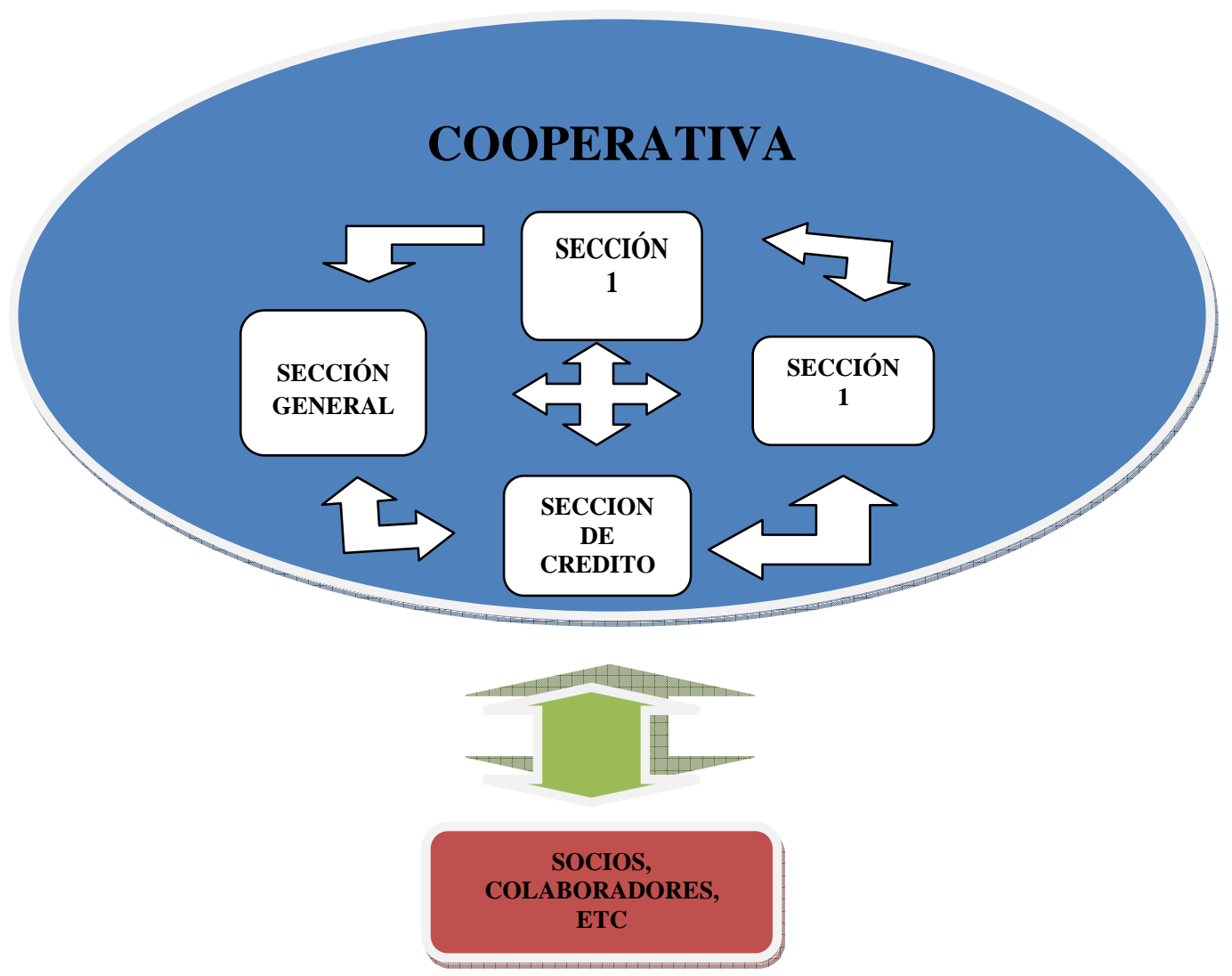

Fuente: Elaboración propia

La legislación de las cooperativas andaluzas, vigente hasta el ejercicio 2011, no contenía referencias específicas al funcionamiento de las secciones de crédito, limitándose a 
regular la obligatoriedad de auditoría de cuentas anuales (Artículo 6.6 Ley 2/1999) y la llevanza de contabilidad independiente y diversos libros de la sección (Artículo 6.5 Ley 2/1999).

Esta ausencia de regulación ha favorecido el florecimiento de las secciones de crédito como instrumento fundamental de apoyo al sector agrario andaluz, en competencia directa con el sector bancario, convirtiéndose en un instrumento de cohesión del cooperativismo y, en muchas ocasiones, como un elemento clave para superar la crisis de precios del sector agrícola. (Campos y otros, 2006).

A finales de 2011, se aprueba la Ley 14/2011, de 23 de diciembre, de sociedades cooperativas andaluzas, limitando a un artículo (artículo 12.5) la referencia a las secciones en general y a las de crédito en particular.

En el desarrollo de la actividad financiera de intermediación, las secciones de crédito han venido realizando operaciones activas y pasivas, de mayor o menor complejidad, obteniendo recursos de unos elementos, básicamente socios, para localizarlos en otros elementos distintos (socios y entidades financieras).

Como veremos más adelante, las operaciones activas son las realizadas por la sección de crédito con sus socios y otras secciones y su objetivo es fomentar la actividad propia de la cooperativa mediante la financiación de éstos. Gráficamente, se representa en el gráfico 2. 
Gráfico 2. Operaciones activas de las secciones de crédito
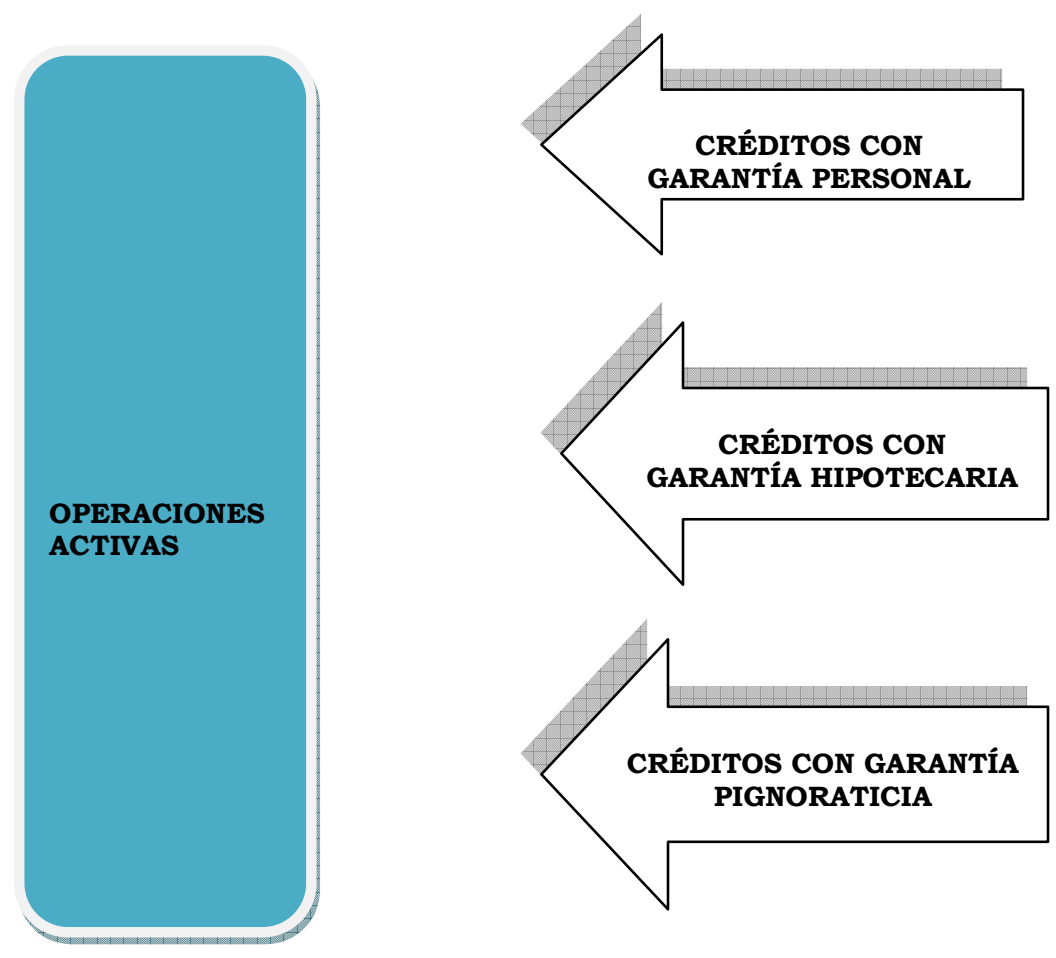

Fuente: Elaboración propia

No debemos olvidar que entre las operaciones activas se encuentra, atendiendo a su finalidad, la financiación del resto de secciones de la cooperativa, constituyéndose en la mayoría de los casos, como los principales inversores de la actividad principal de la cooperativa (corriente o inversiones).

Las operaciones pasivas son las realizadas por la sección de crédito con sus socios destinadas a la captación de recursos en sus distintas formas: a la vista, a plazo o de ahorro. El gráfico 3 muestra los productos más usuales utilizados. 
Gráfico 3. Operaciones pasivas de las entidades de crédito
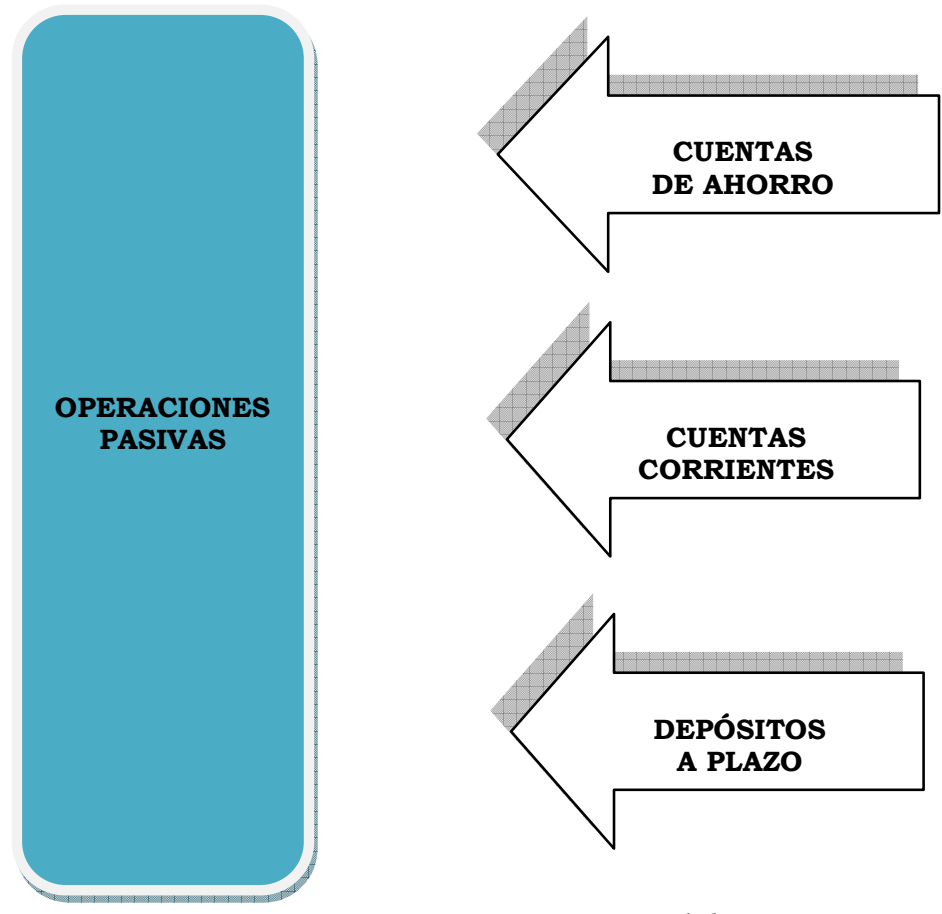

Fuente: Elaboración propia

La captación de recursos cuya finalidad es cubrir las necesidades financieras de socios y otras secciones, puede dar lugar a la generación de excedentes de tesorería cuyo destino es la rentabilización en entidades de crédito, configurándose como operaciones activas de la sección de crédito que contribuyen, mediante su colocación en productos bancarios de distinta naturaleza (depósitos a la vista, a plazo, estructurados, cartera de valores, etc.) y con los límites recogidos en la normativa aplicable, a los objetivos de la misma y, por tanto, de la propia cooperativa.

\section{LA NUEVA REGULACIÓN DE LAS SECCIONES DE CRÉDITO ANDALUZAS}

Establece la Disposición final segunda de la Ley 14/2011, de 23 de diciembre, de Sociedades Cooperativas Andaluzas, que el desarrollo reglamentario de la Ley se llevará a cabo en el plazo de seis meses desde su entrada en vigor dado que, como consecuencia de la nueva ordenación normativa configurada legalmente, sin dicho desarrollo las previsiones contenidas en su texto no podrían desplegar todos sus efectos.

De acuerdo con el mandato legal contenido en la Ley de Cooperativas Andaluzas, se encuentra actualmente en estudio el desarrollo reglamentario de la Ley 14/2011 a través tanto 
de la elaboración de un proyecto de reglamento, como de la toma de datos de campo por parte de la Consejería de Innovación con el objeto de verificar el efecto que pudiera tener la citada normativa en las cooperativas andaluzas.

A fecha de este artículo no ha sido aprobado el Decreto que desarrollará reglamentariamente la citada Ley, si bien, se ha dispuesto de diversos "anticipos" que han culminado en proyectos de decreto que ponen de manifiesto la orientación que la nueva normativa seguirá.

A continuación, se realiza un análisis de la que pudiera ser la propuesta contenida en la normativa a la vista de los proyectos de decreto analizados y la distinta información a que se ha tenido acceso, así como comparación de ésta con la legislación mantenida en otras autonomías.

Dado que el proyecto de reglamento extiende sus efectos a todos los ámbitos de la Ley de Cooperativas Andaluzas cuyo desarrollo e interpretación descansan precisamente en el citado reglamento, nos centraremos exclusivamente en aquellos aspectos del mismo que pudieran tener incidencia exclusivamente en las secciones de crédito.

Conforme al citado proyecto de reglamento, en adelante Reglamento, y el artículo 12 de la Ley 14/2011, de Cooperativas Andaluzas, "se podrán constituir en el seno de la sociedad cooperativa secciones sin personalidad jurídica independiente, para realizar una determinada actividad, siempre que se encuentre comprendida dentro del objeto social de la sociedad cooperativa, ya sea de manera específica o complementaria, y que los estatutos de la entidad incorporen su regulación."

Dentro de estas secciones se incluyen las de crédito que, como el resto, llevarán necesariamente una contabilidad independiente, sin perjuicio de su integración en la contabilidad general de la entidad, quien deberá someter anualmente su estado financiero a auditoría externa.

Pero los aspectos más destacables de la regulación prevista se centran en la regulación económica y financiera de estas secciones, el control de las operaciones con la cooperativa y con los socios y, finalmente, el control externo. 
Para una mejor comprensión del contenido de la normativa andaluza, es necesario realizar una breve descripción de determinados conceptos económicos y financieros asociados a la regulación de las secciones de crédito.

Las operaciones activas

Las operaciones activas realizadas por la sección de crédito se corresponden, desde la perspectiva de este análisis, con los créditos concedidos por la sección de crédito exclusivamente a socios y secciones de la cooperativa. Entre las operaciones activas, se encuentran:

- Anticipos de campaña, con garantía de la aceituna entregada o a entregar.

- Créditos con garantía personal a corto y largo plazo.

- Créditos con garantía real.

- Crédito en cuenta corriente a las secciones.

\section{Excedentes de tesorería}

Se consideran excedentes de tesorería la diferencia neta entre los recursos captados por la sección de crédito con origen en los depósitos de socios, en su distinta instrumentación financiera, y la financiación trasladada al resto de socios y secciones de la cooperativa. Básicamente, es la parte de los depósitos de socios que no se han destinado a operaciones activas (créditos, anticipos, etc.) con los socios y el resto de secciones de la cooperativa.

\section{Las operaciones pasivas}

Las operaciones pasivas realizadas por la sección de crédito se corresponden con la captación de recursos de socios en sus distintas modalidades financieras. Estas operaciones se extienden a los depósitos realizados por otras cooperativas en aquellos casos en que la Sección de crédito se haya constituido en una cooperativa de segundo o ulterior grado, por sus propios socios-cooperativas de base.

\section{Seguridad, Solvencia y liquidez}

Los conceptos de seguridad, solvencia y liquidez a que se refiere el borrador de reglamento en relación con la rentabilización de los excedentes de tesorería de las secciones de crédito en entidades financieras o en otras secciones de crédito en las que la cooperativa se 
encuentra integrada, hay que ponerlos en relación con las inversiones mantenidas por la sección de crédito en entidades financieras con el objetivo de rentabilizar los excedentes de tesorería de la misma.

Estas inversiones deben cumplir con requisitos de:

- Seguridad, en tanto que permitan como mínimo la recuperación del nominal invertido al vencimiento.

- Solvencia, referida a la entidad financiera en la que se colocan estos excedentes.

- Liquidez, en referencia a la capacidad de recuperar en el corto plazo y sin elevados costes, la inversión realizada.

- No obstante, se observa la ausencia de los requisitos anteriores en el resto de operaciones activas de la sección, esto es, operaciones con socios y otras secciones a las que, necesariamente se les debe exigir unos requisitos similares, cuyo cumplimiento debe ser objeto de control y seguimiento.

\section{Activos consolidados}

Debe entenderse al total activo de la cooperativa obtenido de los balances por secciones. En este sentido, la norma contable obliga a elaborar balances por secciones, identificando activos y pasivos imputables a cada una de las secciones.

Este total consolidado, conforme a la estructura económica y financiera de la sección, no tiene por qué coincidir con el total activo de la cooperativa, como consecuencia de la financiación prestada por la sección de crédito al resto de secciones que siendo activo de una, debe ser tratado como pasivo de la receptora de la financiación. No obstante, se trata de un aspecto que, aun estando claro desde la perspectiva económica y financiera, puede plantear alguna duda desde la perspectiva de este reglamento.

\section{Ingresos ordinarios}

Ingresos generados por la actividad corriente de la sociedad cooperativa, incluyendo la actividad principal de fabricación y comercialización de productos, y los ingresos obtenidos por la actividad de la sección de crédito.

Recursos de la sección de crédito 
En el ámbito de esta norma, los recursos propios de la sección de crédito hacen referencia a la financiación disponible para destinarlos a los fines propios de la misma. Entre éstos, se encontrarían los depósitos de socios y otros pasivos asociados a la misma.

\section{ASPECTOS DESTACABLES DE LAS SECCIONES DE CRÉDITO EN LA REGULACIÓN PREVISTA}

Los aspectos cuya regulación, conforme a la orientación escogida por Andalucía, serán objeto de tratamiento en la normativa prevista, se centran en limitar las operaciones activas y pasivas que desarrollarán las secciones de crédito y en incrementar la solvencia y seguridad de gestión y control de las mismas. En concreto,

a) Un capital social mínimo exigible a las sociedades cooperativas con sección de crédito.

En las legislaciones comparativas estudiadas, se exige a las cooperativas con sección de crédito un mínimo de capital social para constituirse, en la legislación extremeña donde es necesario disponer de un capital social mínimo de 3.005,06 € para poder constituir una sección de crédito en el seno de una cooperativa (Decreto-Ley 1/2011, de 11 de noviembre) y en la normativa valenciana (Decreto 99/2007, de 6 de julio) que se exige el establecimiento de un capital social mínimo para obtener y conservar la autorización de $150.000 €$ o disponer de unos recursos propios computables no inferiores a dicha cuantía.

Sin embargo, en la normativa de Cataluña (Decreto 83/2010, de 29 de junio) se determina que los recursos propios de la cooperativa no pueden ser inferiores al 50 por ciento de la suma de los saldos del inmovilizado material e inmaterial neto, una vez deducidos de éstos los saldos de las subvenciones de capital obtenidos para su financiación. Así, como que el total del pasivo exigible de la cooperativa no puede exceder en más de once veces los recursos propios de la cooperativa.

Además, la legislación de Extremadura (Decreto 129/2002, de 24 de septiembre) señala que los depósitos de la sección de crédito no se considerarán recursos propios de la sociedad cooperativa, sino que mantendrán siempre su naturaleza y en consecuencia su exigibilidad frente a terceros.

La Ley 14/2011, de Sociedad Cooperativas Andaluzas y el proyecto de reglamento que desarrolla esta Ley, no prevé importes mínimos de capital social por consecuencia de la Sección de Crédito, estableciendo un mínimo con carácter general. 
b) Limitación al tamaño de la sección de crédito.

La legislación de cooperativas en las comunidades valenciana, catalana, extremeña, y en el proyecto andaluz, fijan límites al tamaño de la sección de crédito en referencia al conjunto de la cooperativa, de manera que no podrá tener una dimensión de tal envergadura que constituya de hecho la actividad principal de la entidad. Es decir, en ningún caso, la actividad principal de las cooperativas con sección de crédito puede ser, precisamente, la "financiera" desarrollada por la sección de crédito. Este planteamiento concuerda con la finalidad última de la sección de crédito de contribuir al objeto social de la cooperativa.

En la prevista legislación andaluza, la actividad de la sección de crédito constituye la actividad principal de la entidad cuando los ingresos ordinarios de la sección excedan de la mitad del resto de los ingresos ordinarios de la entidad durante más de dos ejercicios cerrados consecutivos $y$ cuando su activo total supere en más de un cincuenta por ciento al del conjunto de activos consolidados del balance de la cooperativa.

En este punto parece encontrarse un punto diferenciador favorable en la normativa andaluza respecto a la normativa catalana, extremeña y valenciana. Limitar el tamaño de la sección de crédito en cuanto a volumen, puede significar ahogar de hecho a éstas. Así, en Cataluña se limita a las cooperativas con sección de crédito a invertir en actividades de la cooperativa hasta el 50 por ciento de los recursos de la sección (art. 6 Ley 6/1998).

En Extremadura, las sociedades cooperativas con sección de crédito pueden invertir en actividades de la cooperativa hasta un 30 por ciento de los recursos obtenidos por la propia sección de crédito, pudiendo ampliarse esta cifra hasta un 50 por ciento previo acuerdo (art. 18 Decreto 129/2002).

Finalmente, en Valencia, el volumen de las operaciones de crédito de la sección de crédito deducidos los fondos de insolvencia, no podrá superar, en ningún caso, el 50 por ciento de los recursos propios de la cooperativa (art. 58 Ley 9/2001).

c) Auditar las cuentas anuales y designar una Dirección o Gerencia profesional con dedicación permanente a la gestión de la sección de crédito

Aunque existe homogeneidad en las leyes autonómicas de cooperativas para que la sección de crédito lleve una contabilidad independiente de la general de la cooperativa, no prevalece la misma exigencia con respecto a la auditoría de sus cuentas anuales. Así, en Andalucía, Extremadura (art. 26 Decreto 129/2002) y Valencia (art. 50.1 Ley 8/2003) las cooperativas que dispongan de sección de crédito están obligadas a auditar sus cuentas 
anuales. No obstante, en Cataluña serán verificadas por auditores de cuentas si lo establecen los estatutos o lo acuerda la asamblea general o el consejo rector o lo solicita un número de socios que represente como mínimo el 15 por ciento de los votos sociales de la cooperativa (art 52.1 Ley 18/22).

En cuanto al control interno y profesionalización de la gestión, en las legislaciones de Cataluña (art. 48 Ley 18/2002) y Andalucía se exige un director/a general con facultades específicas y dedicación permanente, mientras que en Extremadura se matiza su obligatoriedad cuando el volumen de depósitos de la sección de crédito supera los 1.500.000 $€$ (art. 14.2 Decreto 129/2002) y en Valencia los 2.404.048 € (art. 1 Orden 16 de enero de 1987).

En Andalucía se prevé que la Dirección o Gerencia profesional, con dedicación permanente a dicha sección, deberá reunir las condiciones de capacidad, preparación técnica y experiencia para desarrollar las funciones propias del cargo.

Adicionalmente, el control externo de las cuentas de la sección se extenderá mediante la emisión de un informe complementario específico referido a la actividad financiera de la sección de crédito, que se elaborará de acuerdo con las normas técnicas dictadas por el Instituto de Contabilidad y Auditoría de Cuentas, y con el contenido mínimo, que en su caso, establezca la Consejería competente en materia de cooperativas. Este informe deberá acompañar al informe de cuentas anuales de la sociedad.

Adicionalmente, las personas auditoras o las sociedades de auditoría de las cuentas anuales de las sociedades cooperativas con sección de crédito estarán obligadas a comunicar por escrito a la Consejería competente en materia de cooperativas, en un plazo máximo de diez días desde el momento en que hayan tenido conocimiento en el ejercicio de sus funciones, cualquier hecho o acuerdo sobre la entidad auditada, que pueda:

- Constituir una violación grave de las disposiciones legales o reglamentarias reguladoras de las secciones de crédito.

- Perjudicar la continuidad de la explotación o, afectar gravemente a la estabilidad o solvencia de la entidad.

- Implicar una opinión desfavorable o denegada, o impedir la emisión del informe de auditoría. 
Entendemos que, a resultas de los posibles incumplimientos que se deriven de la comunicación realizada por el auditor, la potestad inspectora que tiene atribuida la Dirección General competente en materia de cooperativas andaluzas se iniciará de oficio.

d) Limitación de las operaciones con no socios de la sección de crédito.

Las secciones de crédito andaluzas limitarán sus operaciones activas y pasivas a la financiación de las diferentes secciones (con los límites impuestos) y a sus propios socios. Y el excedente de tesorería se podrá rentabilizar a través de entidades de crédito u otras secciones de crédito constituidas en sociedades cooperativas de segundo o ulterior grado en las que las que la cooperativa esté integrada.

Dentro de la actividad propia de la sección de crédito se encuentra la realización de operaciones activas con socios, bien prestando financiación a corto plazo para anticipar el pago de los productos o servicios entregados (anticipos de campaña), bien para financiación a corto o largo plazo bajo la modalidad de préstamos, así como operaciones pasivas mediante la necesaria captación de recursos. Adicionalmente, permite la legislación prevista andaluza la participación limitada de los socios colaboradores (aquellos socios que participan en actividades accesorias de la cooperativa) en la actividad de la sección de crédito pero exclusivamente mediante la realización de operaciones pasivas, esto es, captación de fondos.

Si bien la primera de las financiaciones, anticipos, esta descrita específicamente en la norma, el resto un matiz a considerar. La legislación andaluza permitirá a la sección de crédito conceder préstamos y créditos a los socios cuando contribuyan a la financiación de actividades propias que estén vinculadas a las de la entidad (Ley 14/2011, de 23 de diciembre, de Sociedades Cooperativas Andaluzas), limitando las operaciones de los socios colaboradores con la sección de crédito, a operaciones pasivas.

Por contra, se contempla en la legislación valenciana que las secciones de crédito tienen la probabilidad de efectuar créditos y préstamos a los socios y asociados para cualquier finalidad, exceptuando las operaciones destinadas a inversiones en sectores productivos ajenos a la actividad de la sociedad cooperativa (Decreto 129/2002 de la Comunidad Valenciana).

Más ventajosa, las legislaciones de Extremadura (art. 3.2 Decreto 129/2002) y de Cataluña (art. 1.2 Ley 6/1998) permiten que las secciones de crédito puedan operar con los asociados y colaboradores y se amplía la concesión de crédito y préstamos para el desarrollo de la actividad empresarial y para sus necesidades domésticas. 
e) Volumen de las operaciones activas en la cooperativa.

Como se ha comentado en apartados anteriores, las secciones de crédito deben limitar sus operaciones activas y pasivas al seno de la propia cooperativa. No obstante, es de esperar que los recursos captados de los socios (operaciones pasivas) no se localicen en su totalidad en créditos a socios o al resto de secciones (operaciones activas), pudiendo generarse un excedente de tesorería cuya colocación está sujeta a condiciones, no en cuanto a su importe, sino en cuanto al destino.

Así, este excedente debe destinarse a operaciones en activos de elevada calidad crediticia que garanticen, al menos, la recuperación a su vencimiento del capital invertido y que respondan a criterios suficientes de seguridad, solvencia y liquidez.

En relación con la solvencia y la gestión de los fondos de la sección de crédito, se prevén límites en toda la normativa autonómica a las operaciones activas de la sección de crédito según sean inversiones en corriente o en inmovilizado.

Del importe global invertido en la cooperativa (entiéndase, otras secciones), la normativa autonómica establece límites en relación con la financiación a largo plazo, que pueden ser ampliados en determinadas circunstancias. La financiación del corriente, en cambio, no presenta limitaciones por motivos de liquidez en Cataluña (art. 6 Ley 6/1998), en Extremadura (art. 18.3 Decreto 129/2002) y en Valencia (art. 58 Ley 9/2001).

En Cataluña, el volumen de operaciones activas de la sección de crédito en inversiones de inmovilizado no podrá superar el 25 por ciento de los recursos de la propia sección, no obstante, podrá ampliarse este límite al 50 por ciento por acuerdo expreso en la Asamblea y con el voto favorable de las dos terceras partes de los asistentes (art. 6.2 Ley 6/1998 Cataluña). El resto se podrá destinar a operaciones de crédito (art 6 Ley 6/1998 Cataluña).

En Extremadura, del importe global utilizado por la cooperativa, y previo acuerdo expreso adoptado por la asamblea general, se puede destinar a inversiones de inmovilizado, con carácter transitorio, una cifra no superior al 25 por ciento de los recursos de la sección (art. 18.3 Decreto 129/2002). El resto a inversiones en circulante (art. 18 Decreto 129/2002).

En Valencia, el límite máximo para inversiones en inmovilizado para la cooperativa se fija en el 25 por ciento de los saldos de depósitos en la sección de crédito (art. 8.3 Ley 8/1985). Y el resto a operaciones de crédito (art. 58 Ley 9/2001). 
Finalmente, en la normativa prevista para Andalucía, el importe global invertido en la cooperativa sólo se podrá destinar a inversiones de inmovilizado una cifra no superior al 25 por ciento de los recursos de la sección de crédito. Adicionalmente, el volumen de las operaciones activas de crédito no podrá superar el 50 por ciento de los recursos de la propia sección de crédito, salvo operaciones con la cooperativa, en cuyo caso podrá incrementarse por acuerdo de la asamblea general hasta un máximo del 75 por ciento para anticipos a los socios y con un plazo de devolución no superior al año. Entendemos que la normativa andaluza pretende aportar una garantía adicional a los recursos de la sección limitando la inversión de éstos en la propia cooperativa, planteamiento éste bastante acertado puesto que su implantación va a poner de manifiesto situaciones de "autofinanciación" probablemente llamativas y en algunos casos escasamente conocidas por los propios socios.

Es de destacar que, en el supuesto de que la sección financie el inmovilizado material de otras secciones, los activos financiados no podrán ser objeto de garantía o pignoración.

f) Excedente de tesorería y coeficiente de disponibilidades líquidas

Prevé la normativa andaluza que las secciones de crédito sólo podrán colocar sus excedentes de tesorería en productos de entidades financieras y secciones de crédito que aseguren, como mínimo, la recuperación del capital invertido a su vencimiento y que respondan a criterios suficientes de seguridad, solvencia y liquidez.

En Cataluña, no se limita de forma explícita la colocación de los excedentes de tesorería, expresando que se deberán gestionar conjuntamente las disponibilidades líquidas de los socios y de los adheridos (art. 1.2 Ley 6/1998).

Sin embargo en la legislación de Extremadura (art.81.1 Ley 5/2001) y de Valencia (art. 1 Ley 8/1985 y Decreto 129/2002) se contempla, para una mejor gestión de los fondos, que los excedentes de tesorería se podrán colocar en depósitos de otros intermediarios financieros, fondos públicos y valores emitidos por empresas públicas, y se añade en la normativa extremeña la obligatoriedad de que la actividad de estas empresas se ejerza con preferencia en el territorio de su comunidad autónoma.

Para asegurar la liquidez de la sección de crédito, en la legislación valenciana se determina que habrá de mantener un coeficiente de disponibilidades líquidas (Decreto 129/2002), pudiendo la Consejería de Economía, Industria y Comercio modificar dicho coeficiente en función de las circunstancias del momento pero no podrá ser inferior al 10 
por ciento del volumen de depósitos de la sección de crédito. Dicho coeficiente, cuyo cumplimiento correcto requiere una observancia diaria, se computará tomando como denominador el volumen de depósitos de la sección de crédito y como numerador el efectivo de la caja más los saldos mantenidos a este fin, en entidades de crédito.

En la normativa de Extremadura el coeficiente de disponibilidades líquidas que habrán de mantener las cooperativas con sección de crédito para asegurar la liquidez de dicha sección no podrá ser inferior al 10 por ciento (art. 20 Decreto 151/1986) del volumen de depósitos de la sección de crédito.

En Andalucía, la legislación prevista sólo especifica que deberá mantenerse un coeficiente de disponibilidades líquidas que no podrá ser inferior al 15 por ciento del volumen de depósitos de la sección de crédito.

En la legislación de Cataluña será la asamblea general la que fijará el porcentaje de disponibilidades líquidas que mantendrá la sección de crédito en relación con sus recursos (art. 8 Ley 6/1998).

g) Operaciones activas de la Sección de Crédito con otras secciones: coste de la financiación.

La Sección de Crédito se ha convertido, en los últimos años y para muchas sociedades cooperativas, en un financiador de bajo coste para cubrir las importantes inversiones que la evolución del sector ha requerido. En muchos casos, esta financiación del inmovilizado ha sido "gratuita" o a un coste muy reducido.

El coste de la financiación prestada por la sección de crédito a la cooperativa, entiéndase resto de secciones, se referencia en la distinta normativa autonómica al interés legal del dinero o al Euribor. Así, en caso de la legislación extremeña, este coste no podrá ser inferior al interés legal del dinero, (art. 18.1 y 2 Decreto 129/2002).

La legislación valenciana prevé que la retribución sea igual al Euribor a un año más un punto (art. 17 Ley 14/1997). Sin embargo, la legislación catalana establece que el consejo rector, con un informe previo del director o gerente a cada operación crediticia y que constará en acta, establecerá los intereses a imputar a favor de la sección (art. 6.1 Ley 6/1998).

En la normativa andaluza se especifica que las condiciones económicas aplicables a las operaciones activas y pasivas se ofrecerán en términos de no discriminación a los socios y serán públicas (tipos de interés, comisiones y gastos). En relación con las 
operaciones crediticias con la propia sociedad cooperativa, el tipo de interés establecido no podrá resultar inferior al interés legal del dinero.

h) Limitación a las operaciones de riesgo a un socio o unidad de riesgo.

No sólo se regula la naturaleza de las operaciones de financiación, limitándolas a aquellas vinculadas con su actividad, sino que se establece un límite a la concentración de riesgos, de manera que no se pueden conceder operaciones a un socio o a una unidad de riesgo en un porcentaje del 2,5 por ciento de los recursos totales de la cooperativa, tanto en la legislación de Cataluña (art. 6.1 Decreto 280/2003) y como en la normativa andaluza.

En este punto es importante que la norma defina el concepto de unidad de riesgo, como a los titulares de operaciones de riesgo que tengan una relación de consanguinidad o afinidad en primer grado, así como aquellos que conjuntamente destinen los préstamos o créditos recibidos a la misma aplicación, o aporten la misma garantía. Si a criterio de la Dirección o Gerencia, dos o más personas puedan considerarse, en función de su independencia económica, que no son unidad de riesgo podrán exceptuar de este tratamiento.

Extremadura señala que no podrá prestar más del 5 por ciento de los depósitos a un solo socio o asociado o a un grupo de socios que constituyan una unidad de riesgo (art.19.2 Decreto 129/2002).

Y en Valencia, la legislación coincide con Extremadura pero matiza que excepcionalmente la Consellería podrá autorizar operaciones que superen el límite de riesgo establecido sin exceder, en ningún caso, del 10 por ciento (art. 9.2 Ley 8/1985) (Decreto 129/2002).

Finalmente, las secciones de crédito no podrán instrumentar riesgos de firma con socios ni adheridos, según la normativa catalana (art. 7.3 Ley 6/1998), ni con socios ni asociados en la legislación extremeña (art. 19.4 Decreto 129/2002), sólo en la normativa valenciana se asume que el nivel máximo de riesgos de firma con socios y asociados no podrá superar el volumen de recursos propios de la cooperativa (art. 9.4 Ley 8/1985) (Decreto 129/2002).

Dado que el tejido societario de las cooperativas agrarias andaluzas está formado por muchas comunidades de bienes, que agrupan a personas con relación de afinidad o consanguinidad, el límite previsto en la normativa autonómica andaluza se puede plantear que el mismo se supere con cierta facilidad, pudiendo ser solventado de alguna manera 
incorporando garantías reales a los préstamos concedidos lo que supondría un aumento de la seguridad de las operaciones activas de la sección de crédito con sus socios.

i) Limitación en las operaciones con socios miembros del Consejo Rector.

Por la trascendencia de las operaciones con partes vinculadas, éstas son objeto de tratamiento diferenciado, de manera que las operaciones activas con miembros del Consejo Rector o cualquier otro miembro de órganos ejecutivos, así como aquellas que guarden relación de parentesco de consanguinidad o afinidad hasta el segundo grado, requerirán:

- Acuerdo del órgano de administración en el que no participará la persona miembro afectada, al considerarse conflicto de intereses.

- Si se trata de administración única, deberá ser objeto de aprobación por asamblea general.

\section{CONCLUSIONES}

El panorama a corto plazo para las secciones de crédito andaluzas es tranquilizador, si bien no está libre de tensiones que exigirán el máximo interés y dedicación de sus componentes, de las organizaciones que defienden los intereses de las cooperativas ${ }^{4}$ y de las Administraciones Públicas.

El esfuerzo realizado en los últimos años para generar confianza, mejorar las expectativas de sus socios, favorecer el desarrollo rural y consolidar el sector agrario, no se va a ver mermado por unos mecanismos de control y profesionalidad del sector, sino todo lo contrario.

Los distintos episodios vividos en los últimos años de crisis merecen ser interpretados convenientemente, y orientar la legislación hacia una mejora en la gestión y control de las secciones de crédito. Es cierto que se han producido situaciones anómalas en diversas secciones de crédito, también es cierto que la incorrecta gestión de las secciones de crédito ha podido provocar desequilibrios patrimoniales que han repercutido finalmente en los socios, asociados, etc., pero no es menos cierto que siendo el objetivo último de las secciones la contribución al objeto de la cooperativa, sin que se constituya su actividad en un fin en sí mismas, los riesgos se encuentran más localizados y, con la nueva regulación andaluza se contribuirá a la reducción de éstos. La experiencia nos dice que es necesario establecer mecanismos de control y profesionalizar el sector en su vertiente financiera. El volumen de

\footnotetext{
${ }^{4}$ Como la Federación Andaluza de Empresas Cooperativas Agrarias (FAECA)
} 
depósitos que las secciones pueden llegar a controlar y la avidez con que otros operadores financieros observan, exigen un adecuado control y seguimiento de las operaciones realizadas por las secciones de crédito, extendiendo éste control a la concesión de créditos a los miembros de cualquier órgano ejecutivo, directores y sus familiares, mediante la previa autorización del órgano de administración o asamblea general en caso de administrador único.

La regulación de las operaciones activas y pasivas, sin llegar a ahogar la operativa de las secciones de crédito, permitirán establecer límites que facilitaran la solvencia de estas entidades, lo que unido a una gestión profesional, que no tiene que venir de la mano de un gerente profesional, y a un control externo más amplio, asegurarán la supervivencia de las secciones de crédito y reforzarán la confianza en estos intermediarios entre el mundo cooperativo y el financiero.

El proyecto de normativa analizado plantea grandes y serios retos a las secciones de crédito andaluzas en los términos que algunas de ellas se han venido desarrollando y funcionando en los últimos años. El mayor coste de la profesionalización y control externo, se verá suficientemente compensado con una mayor solvencia de las secciones de crédito y un aumento de seguridad y confianza de los agentes intervinientes (socios y entidades financieras) en las operaciones activas y pasivas.

El efecto a corto plazo que la normativa prevista pudiera tener en las secciones de crédito, podría determinarse empíricamente mediante un estudio limitado a varias cooperativas de Andalucía, analizando aquellos aspectos que no se han podido concretar en este informe teórico al objeto de identificar riesgos y planificar soluciones, no obstante, resulta imprescindible un profundo estudio de la actual situación de las secciones de crédito andaluzas, estructura financiera de las mismas, servicios prestados, destinatarios de los mismos y, en definitiva, un análisis de la realidad de éstas en el cooperativismo andaluz.

Finalmente, la normativa andaluza constituye un paso adelante hacia la regulación y control de las secciones de crédito en Andalucía, apostando por su continuidad en un marco de control más efectivo pero sin limitar, como otras comunidades, su desarrollo pudiera ser imitado en otras comunidades que aún no han desarrollado su propia normativa. 


\section{BIBLIOGRAFÍA}

BEL DURÁN, P (2000) La financiación de las sociedades cooperativas agrarias con sección de crédito. REVESCO. Revista de Estudios Cooperativos [en línea] 2000: [fecha de consulta: 12 de abril de 2013] Disponible en: <http://www.redalyc.org/articulo.oa?id=36707201> ISSN 1135-6618

CAMPOS CLIMENT, V; FAJARDO GARCÍA, G y SANCHÍS PALACIO, J.R. (2006) Triple justificación de las secciones de crédito de las cooperativas agrarias de la comunidad valenciana: jurídica, económica y social. CIRIEC-España, Revista de Economía Pública, Social y Cooperativa, abril, nº 054, p. 129-165.

CAMPOS CLIMENT, V y SANCHÍS PALACIO, J.R. (2005) Las secciones de crédito de las cooperativas en España. Análisis de su situación actual y su papel en la financiación al cooperativismo agrario y al desarrollo rural. Revista de Desarrollo Rural y Cooperativismo Agrario, n $^{\circ}$ 9, p. 35-52.

GARCÍA-GUTIERREZ, C. (1992) Análisis de la rentabilidad financiera y económica de los socios de las cooperativas: la influencia de una rentabilidad en la otra y la aplicación del criterio (principio) de justicia -que no de solidaridad- en la distribución de la ganancia real". Revista Europea de Dirección y Economía de la Empresa, Vol. 1, nº 2, p. 115-124.

MELIÁN NAVARRO, A. (2005) Divergencias legislativas de las secciones de crédito de las cooperativas en España. CIRIEC- España, Revista de Economía Pública, Social y Cooperativa, abril nº 051, p. 131-166.

\section{Disposiciones legales o normativas}

Estado:

LEY 27/1999, de 16 de julio, de Cooperativas (BOE Nº170, de 17 de julio).

ORDEN EHA/3360/2010, de 21 de diciembre (RCL 2010, 3308 y RCL 2011,162) (BOE N 316 de 29 de diciembre de 2010)

Andalucía:

LEY 2/1999, de 31 de marzo, de Sociedades Cooperativas Andaluzas (BOJA No 46, de 20 abril).

LEY 3/2002, de 16 de diciembre por la que se modifica la Ley 2/1999, de 31 de marzo de Sociedades Cooperativas Andaluzas (BOJA No 153 de 28 de diciembre de 2002) (BOE $\mathrm{N}^{\mathrm{o}} 10$, de 11 de enero de 2003). 
LEY 14/2011, de 23 de diciembre, de Sociedades Cooperativas Andaluzas (BOJA No 255, de 31 de diciembre).

Cataluña:

LEY 6/1998, de 13 de mayo, de Regulación del Funcionamiento de las Secciones de Crédito de las Cooperativas. (DOGC No 2644, de 21 de mayo de 1998).

LEY 18/2002, de 5 de julio de cooperativas (DOGC No 3679, de 17 de julio de 2002 y BOE $\mathrm{n}^{\circ} 179$, de 27 de julio de 2002)

LEY 13/2003, de 13 de junio, de modificación de la Ley 18/2002, de 5 de julio, de Cooperativas (BOE $\mathrm{n}^{\circ} 171$, de 18 de julio).

DECRETO 280/2003, de 4 de noviembre, de desarrollo de la Ley 6/1998, de 13 de mayo, de regulación del funcionamiento de las secciones de crédito de las cooperativas (DOGC N ${ }^{\circ}$ 4014, de 20 de noviembre).

DECRETO 83/2010, de 29 de junio, por el que se modifica el Decreto 280/2002, de 4 de noviembre, de regulación del funcionamiento de las secciones de crédito de las cooperativas (DOGC N 5664 de 06 de julio de 2010).

DECRETO-LEY 1/2011, de 15 de febrero de modificación de la Ley 18/2002, de 5 de julio, de cooperativas de Cataluña (BOE nº 76, de 30 de marzo de 2011).

Extremadura:

DECRETO 129/2002, de 24 de septiembre, que regula el funcionamiento de las secciones de crédito de las sociedades cooperativas en la Comunidad Autónoma de Extremadura (DOE de 1 de octubre de 2002).

DECRETO 172/2002, de 17 de diciembre por el que se aprueba el Reglamento del Registro de Sociedades Cooperativas de Extremadura y se modifica el art. 21 del Decreto 129/2002, de 24 de septiembre, por el que se regula el funcionamiento de las Secciones de Crédito de las Sociedades Cooperativas de la Comunidad Autónoma de Extremadura (DOE $\mathrm{n}^{\circ}$ 152, 31 de diciembre 2002).

LEY 5/2001, de 10 de mayo, de Crédito Cooperativo (BOE № 164, de 10 de julio)

DECRETO-LEY 1/2011, de 11 de noviembre, por el que se modifica la Ley 2/1998, de 26 de marzo, de Sociedades Cooperativas de Extremadura (BOE N 313, de 29 de diciembre).

Comunidad Valenciana:

LEY 8/1985, de 31 de mayo, de Regulación de la actuación financiera de las Cooperativas con Sección de Crédito de la Comunidad Valenciana (DOGV № 259, de 10 de junio de 1985). 
DECRETO 151/1986, de 9 de diciembre, por el que se desarrolla la Ley 8/1995, de 31 de mayo, sobre Cooperativas con Sección de Crédito (DOCV nº 496 de 30 de diciembre de 1986).

ORDEN de 16 de enero 1987, de la Consellería de Economía y Hacienda sobre Secciones de Crédito de Cooperativas (DOGV de 30 de enero de 1987).

ORDEN de 23 de junio de 1997, de la Consellería de Economía y Hacienda y Administración Pública, por la que se aprueban las bases reguladoras del Plan de Racionalización de las cooperativas con sección de crédito para 1997 (DOGV de 28 de agosto de 1997).

LEY 14/1997, de 26 de diciembre, de Medidas de Gestión Administrativa y Financiera y de Organización de la Generalitat (DOGV no 3153, de 31 de diciembre de 1997).

ORDEN 22 de junio de 1998, de la Consellería de Economía y Hacienda y Administración Pública, por la que se aprueban las bases reguladoras del Plan de Racionalización de las cooperativas con sección e crédito para 1998 (DOGV Nº 3274, de 29 de junio de 1998).

ORDEN de 14 de septiembre de 1999, de la Consellería de Economía y Hacienda y Administración Pública, por la que se aprueban las bases reguladoras del Plan de Racionalización de las cooperativas con sección de crédito para 1999 (DOGV Nº 3590, de 24 de septiembre).

ORDEN de 25 de septiembre de 2000, de la Consellería de Economía y Hacienda y Administración Pública, por la que se aprueban las bases reguladoras del Plan de Racionalización de las cooperativas con sección de crédito para 2000 (DOGV No 3848, de 2 de octubre).

ORDEN de 16 de octubre de 2001, de la Consellería de Economía y Hacienda y Empleo, por la que se aprueban las bases reguladoras del Plan de Racionalización de las cooperativas con sección de crédito para 2001 (DOGV Nº 4113, de 24 de octubre).

LEY 9/2001, de 27 de diciembre, de Medidas de Gestión Administrativa y Financiera y de Organización de la Generalitat Valenciana (DOGV N 4158, de 31 de diciembre de 2001, corrección de errores en DOGV No 4196, de 22 de febrero de 2002).

ORDEN DE 19 de junio, de 2002, de la Consellería de Economía y Empleo, por la que se aprueban las bases reguladoras del Plan de Racionalización de las cooperativas con sección de crédito para 2001 (DOGV Nº 4279, de 26 de junio).

LEY 8/2003, de 24 de marzo, de Cooperativas de la Comunidad Valenciana (DOGV N 4468, de 27 de marzo, BOE No 87, de 11 de abril). 